\title{
Specific Imaging of Bacterial Infection Using 6" -18F-Fluoromaltotriose: A Second-Generation PET Tracer Targeting the Maltodextrin Transporter in Bacteria
}

\author{
Gayatri Gowrishankar ${ }^{1}$, Jonathan Hardy ${ }^{2}$, Mirwais Wardak*1, Mohammad Namavari*1, Robert E. Reeves ${ }^{3}$, \\ Evgenios Neofytou ${ }^{4}$, Ananth Srinivasan ${ }^{1}$, Joseph C. Wu ${ }^{1,4}$, Christopher H. Contag ${ }^{2}$, and Sanjiv Sam Gambhir ${ }^{1,5}$ \\ ${ }^{I}$ Department of Radiology, Stanford University School of Medicine, Stanford, California; ${ }^{2}$ Department of Pediatrics, Stanford \\ University School of Medicine, Stanford, California; ${ }^{3}$ Thermo Fisher Scientific, Hanover Park, Illinois; ${ }^{4}$ Stanford Cardiovascular \\ Institute, Stanford University School of Medicine, Stanford, California; and ${ }^{5}$ Department of Bioengineering, Stanford University \\ School of Medicine, Stanford, California
}

$6 "$ - ${ }^{18} \mathrm{~F}$-fluoromaltotriose is a PET tracer that can potentially be used to image and localize most bacterial infections, much like ${ }^{18} \mathrm{~F}-\mathrm{FDG}$ has been used to image and localize most cancers. However, unlike ${ }^{18} \mathrm{~F}-\mathrm{FDG}, 6^{\prime \prime}-{ }^{18} \mathrm{~F}$-fluoromaltotriose is not taken up by inflammatory lesions and appears to be specific to bacterial infections by targeting the maltodextrin transporter that is expressed in gram-positive and

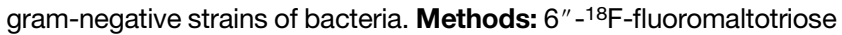
was synthesized with high radiochemical purity and evaluated in several clinically relevant bacterial strains in cultures and in living mice. Results: 6 " - ${ }^{18} \mathrm{~F}$-fluoromaltotriose was taken up in both gram-positive and gram-negative bacterial strains. $6 "-18 \mathrm{~F}$-fluoromaltotriose was also able to detect Pseudomonas aeruginosa in a clinically relevant mouse model of wound infection. The utility of $6^{\prime \prime}-18$ F-fluoromaltotriose to help monitor antibiotic therapies was also evaluated in rats. Conclusion: 6 " - ${ }^{18} \mathrm{~F}$-fluoromaltotriose is a promising new tracer that has significant diagnostic utility, with the potential to change the clinical management of patients with infectious diseases of bacterial origin.

Key Words: infection imaging; bacteria; 6 "-18 F-fluoromaltotriose; PET/CT imaging

J Nucl Med 2017; 58:1679-1684

DOI: 10.2967/jnumed.117.191452

\footnotetext{
A

ntimicrobial resistance as defined by the World Health Organization is the "ability of bacteria to change such that the current regimen of antibiotics becomes ineffective" (1). In 2015, there were 480,000 new cases of multidrug-resistant tuberculosis (2). A high proportion of hospital-acquired infections are now attributed to methicillin-resistant Staphylococcus aureus and other resistant forms of Escherichia coli. A recent report from the Centers for Disease Control and Prevention discussed this emerging public health crisis and estimated that antibiotic resistance added about $\$ 20$ billion in excess direct health-care costs, not including the additional costs to

Received Feb. 7, 2017; revision accepted Apr. 25, 2017.

For correspondence or reprints contact: Sanjiv Sam Gambhir, Department of Radiology, Stanford University School of Medicine, 318 Campus Dr., Stanford, CA 94305

E-mail: sgambhir@stanford.edu

${ }^{\star}$ Contributed equally to this work.

Published online May 10, 2017.

COPYRIGHT (c) 2017 by the Society of Nuclear Medicine and Molecular Imaging
}

society in terms of lost productivity $(3,4)$. Among the many measures advocated by the Centers for Disease Control and Prevention to combat this crisis, there was a call for the development of novel diagnostics to detect and therefore help manage the treatment of infectious diseases. The traditional approach to diagnosing a bacterial infection involves biopsy of the infected tissue or blood and culture of the pathogenic microorganisms in defined microbiologic medium. Advances in bacterial genomics and 16s ribosomal RNA sequencing have bolstered this approach and improved our ability to identify the nature of the pathogen causing the disease (5).

Imaging, particularly nuclear medicine, has always played an important role in the diagnosis of infectious diseases. The use of ${ }^{67} \mathrm{Ga}$-citrate, ${ }^{18} \mathrm{~F}-\mathrm{FDG}$, and labeled leukocytes has been well documented (6-8). Unfortunately, none of these tracers are specific for bacteria and are therefore unable to distinguish bacterial infection from sterile inflammation. Recent years have seen the development of several PET tracers, including ${ }^{68} \mathrm{Ga}$-ubiquicidin and 2 '-deoxy-2'fluoro-1 $\beta$ D-arabinofuranosyl-5- ${ }^{124}$ I-iodouracil ( ${ }^{124}$ I-FIAU) (9-11). Of these, ${ }^{68} \mathrm{Ga}$-ubiquicidin has shown promise in preclinical studies (11), but further evaluation in multicenter clinical trials is warranted $(11,12)$. Other tracers including ${ }^{18} \mathrm{~F}$-fluorodeoxysorbitol have been shown to detect only a specific class of bacteria (e.g., gram-negative Enterobacteriaceae) (13). We have previously demonstrated the development of $6{ }^{18} \mathrm{~F}$-fluoromaltose (Supplemental Fig. 1; supplemental materials are available at http://jnm.snmjournals.org), a probe from a novel class of tracers targeting the maltodextrin transporter in bacteria $(14,15)$. The concept of this class of tracers stems from an earlier study by Murthy et al., who showed that the maltodextrin transporter is unique to bacteria and that a fluorescently labeled maltohexose probe was able to image $E$. coli-induced myositis in rats (16). The maltose-maltodextrin transporter belongs to the adenosine triphosphate (ATP)-binding cassette (ABC) family of transporters. Extensively studied in E. coli, this transporter system has been shown to be present in many bacterial species, both gram-positive and gram-negative, including Enterococcus faecalis, Staphylococcus aureus, Streptococcus pneumoniae, Bacillus subtilis, Listeria monocytogenes, and Vibrio cholera (17). The maltodextrin transporter has also been implicated in the virulence mechanisms of some of these pathogens (18-21). The transporter is a complex of 5 proteins with the outer membrane maltoporin (LamB) and the periplasmic maltose binding protein (MalE) serving as the key determinants of transporter specificity (17). The 
transporter appears to be somewhat promiscuous and has been reported to take up a wide variety of maltose analogs, which has benefited tracer design (22).

Murthy et al. reported the development of a tracer based on the ${ }^{18} \mathrm{~F}$-labeled maltohexose $\left(\mathrm{MH}^{18} \mathrm{~F}\right)$ (Supplemental Fig. 1) (23). Both our prior study and the work from Murthy et al. demonstrated that this class of tracers was not only specific for bacteria but also could accurately distinguish infection from sterile inflammation. However, both $6-{ }^{18} \mathrm{~F}$-fluoromaltose and $\mathrm{MH}^{18} \mathrm{~F}$ suffered from suboptimal pharmacokinetics and had poor signal-to-noise ratios, particularly in the thoracic region, which would preclude the use of these tracers for many clinical indications including lung and cardiac infections. In an effort to clinically translate this class of tracers, we have developed an improved second-generation tracer, $6 "-{ }^{18} \mathrm{~F}$-fluoromaltotriose, building on the same scaffold. The trisaccharide maltotrioses are also substrates for the maltodextrin transporter and are implicated in the virulence of bacterial pathogens including Group A Streptococcus (24). In this study, we demonstrate that $6 "-{ }^{18} \mathrm{~F}$-fluoromaltotriose is taken up by a variety of pathogenic bacterial strains in vitro and in vivo and that its pharmacokinetic profile is superior to the previously characterized probes for the maltodextrin transporter.

\section{MATERIALS AND METHODS}

\section{Synthesis}

6 " -deoxy-6" - ${ }^{18}$ F-fluoro- $\alpha$-D-glucopyranosyl-(1-4)-O- $\alpha$-D-glucopyranosyl-(1-4)-O-D-glucopyranoside $\left(6 "{ }^{\prime \prime}{ }^{18} \mathrm{~F}\right.$-fluoromaltotriose) was prepared by nucleophilic displacement of the nosylate group in $2 ", 3$ ", 4" -tri-O-acetyl-6" -deoxy-6" -O-nosyl- $\alpha$-D-glucopyranosyl(1-4)-O-(2', 3', 6' -tri-O-acetyl- $\alpha$-D-glucopyranosyl-(1-4)-1,2,3,6 tetra$\mathrm{O}$-acetyl-D-glucopyranoside precursor by ${ }^{18} \mathrm{~F}$-fluoride ion in dimethyl formamide $(1 \mathrm{~mL})$ at $85^{\circ} \mathrm{C}$ for $10 \mathrm{~min}$. After being cooled to room temperature, $10 \mathrm{~mL}$ of water were added and the solution passed through a light C-18 Sep-pack cartridge (Water) and the crude protected $6^{\prime \prime}-18 \mathrm{~F}$-fluoromaltotriose removed by passing $3 \mathrm{~mL}$ of acetonitrile through the cartridge. The crude protected $6 "{ }^{18} \mathrm{~F}$-fluoromaltotriose was concentrated and deprotected first by $1 \mathrm{~N} \mathrm{HCl}(1 \mathrm{~mL})$ at $110^{\circ} \mathrm{C}$ for $10 \mathrm{~min}$ and then by $2 \mathrm{~N} \mathrm{NaOH}(0.5 \mathrm{~mL})$ in room temperature for 5 min to afford crude $6 "{ }^{18} \mathrm{~F}$-fluoromaltotriose. After neutralization and high-performance liquid chromatography purification of the solution, $6 "{ }^{18} \mathrm{~F}$-fluoromaltotriose was recovered in $6 \%-9 \%$ radiochemical yield (decay-corrected) with the $95 \%$ radiochemical purity.

\section{Cultures}

E. coli was obtained from American Type Culture Collections (ATCC 33456). The bioluminescent strain of Pseudomonas aeruginosa (Xen 5) and S. aureus (Xen 36) were obtained from Perkin Elmer. The mammalian cell lines HCC1806 and HCC827 (breast and lung cancer cell lines, respectively) were obtained from ATCC and were grown in medium recommended by ATCC.

\section{Bacterial Uptake Studies}

Bacterial uptake studies were performed as described in our previous publication (14). In brief, an overnight $(\mathrm{O} / \mathrm{N})$ culture of the respective strain of bacteria was initiated by inoculating a colony from a plate into a 3-mL culture of Luria-Bertani (LB) broth. The next morning, $500 \mu \mathrm{L}$ of the $\mathrm{O} / \mathrm{N}$ culture were inoculated into $30 \mathrm{~mL}$ of $\mathrm{LB}$ in a $200-\mathrm{mL}$ flask and grown in a $37^{\circ} \mathrm{C}$ shaker/incubator until the bacterial culture reached $\log$ phase $\left(\mathrm{OD}_{600}=0.5\right)$. Aliquots of $10^{8}$ colony-forming units (CFUs) of the bacterial culture were incubated with $0.37 \mathrm{MBq}$ of the tracer for the designated periods. At the end of the incubation period, unbound tracer was removed by washing, and the cultures were lysed using a bacterial lysis solution (BugBuster; EMD).
The counts associated with the lysate were determined using a $\gamma$-counter. The protein concentration in the lysate was determined using a bicinchoninic acid assay (BCA assay) (Pierce, Thermo Fisher Scientific). All samples were compared with total activity references, and the percentage uptake per microgram of protein was calculated.

\section{E. Coli-Induced Murine Myositis}

All animal models were approved by the Stanford University Institutional Animal Care and Use Committee. Nude mice $(n=4$; 6-7 wk old) were anesthetized by isoflurane inhalation. A specific strain of E. coli (ATCC 33456) $\left(1 \times 10^{8}\right.$ CFUs) in $50 \mu \mathrm{L}$ of LB broth was administered, as an intramuscular injection, into the right thigh of the mice as described previously (14). The mice were imaged $24 \mathrm{~h}$ after the initial infection.

\section{P. Aeruginosa Wound Infection Model}

CD1 mice ( $n=8 ; 8$ wk old) were anesthetized by isoflurane inhalation. A small wound was made on the back of the mice using a sharp pair of scissors. Xen 5 ( $\left.10^{6} \mathrm{CFUs}\right)$, a bioluminescent strain of P. aeruginosa, in $20 \mu \mathrm{L}$ of saline was inoculated into a small pocket just under the skin beneath the wound. The wound was then sealed with Vetbond (1469SB; 3M) adhesive. The mice were imaged $24 \mathrm{~h}$ after the infection.

\section{Monocytogenes Model}

Eight-week-old CD1 mice ( $n=5$ ) were infected intravenously with $2 \times 10^{5}$ CFUs of the bioluminescent $L$. monocytogenes strain Xen 32 . Bioluminescence imaging was performed the next day as described above. The mice were imaged laterally on the left side to reveal splenic signal characteristic of listeriosis in CD1 mice. At this dose, the infection is lethal in $3-5 \mathrm{~d}$.

\section{Lipopolysaccharide (LPS) Lung Inflammation Model}

BALB/c mice (6-7 wk old) were anesthetized by isoflurane inhalation. LPS (from E. coli serotype 0127:B8; Sigma) $(50 \mu \mathrm{g})$ in $20 \mu \mathrm{L}$ of saline $(0.9 \% \mathrm{NaCl})$ was administered via intranasal instillation. The control group consisted of mice without any treatment because simple administration of vehicle (saline) is enough to induce lung inflammation. Twenty-four hours after LPS administration, the mice were sacrificed for biodistribution studies and the lungs were saved for histopathologic analysis.

\section{Heat Inactivation of $E$. coli}

An $\mathrm{O} / \mathrm{N}$ culture of the same strain of $E$. coli was set up as described above and allowed to reach $\log$ phase. Aliquots of the culture containing $10^{6}, 10^{7}$, or $10^{8} \mathrm{CFUs}$ of bacteria were heat inactivated at $90^{\circ} \mathrm{C}$ for $30 \mathrm{~min}$ and then implanted in the left thigh of nude mice $(n=3)$. An equal number of viable bacteria were implanted in the contralateral thigh by intramuscular injection. One hour later, the mice were imaged by small-animal PET/CT.

\section{Bioluminescence Imaging}

In vivo bioluminescence imaging was performed using the IVIS Spectrum instrument (Perkin Elmer). The engineered bioluminescent strains of bacteria are able to synthesize their own substrate for the bacterial luciferase, so there was no need to administer substrate. The mice were positioned in the instrument after being anesthetized with isoflurane and imaged under large binning conditions for a suitable exposure time (up to $5 \mathrm{~min}$ ).

\section{Small-Animal PET/CT}

The radiotracer $(7.4 \mathrm{MBq})$ was administered to the mice intravenously as described previously (14). The mice were kept anesthetized with isoflurane after tracer administration. At the desired times, the mice were placed on the bed of the small-animal PET/CT scanner (Inveon; Siemens), and 5-min static scans were obtained. For the 
dynamic scans, tail vein catheters (12-cm polyurethane tubing and 27-g butterfly needle) were inserted and the catheter was glued onto the tail using Vetbond (tissue glue). Once the animal was in position in the PET part of the PET/CT scanner, 7.4 MBq of the tracer were administered via the catheter and PET scanning was started. Dynamic scanning was performed for an hour. During the scan, special precautions were taken to ensure the mice were warm. All images were reconstructed using 3-dimensional ordered-subset expectation maximization. Region-of-interest analysis was performed using IRW software (Inveon Research Workplace; Siemens). No partial-volume corrections were performed on the PET image data.

\section{Biodistribution Studies}

The radiotracer $(7.4 \mathrm{MBq})$ was administered to the rodents intravenously. The rodents were kept anesthetized after tracer administration. At the desired time after tracer injection, the mice were sacrificed by cervical dislocation. Then relevant organs and tissues were removed, placed in $\gamma$-counter tubes, and weighed. Tissue-associated radioactivity was determined in a $\gamma$-counter (Cobra; Perkin Elmer), decay-corrected to time of tracer injection, and normalized to total injected activity using diluted aliquots of the initial administered dose as standards as described previously (14).

\section{Histology}

The tissues were collected in formalin, embedded in paraffin, and processed. The lung sections were stained with hematoxylin and eosin using standard protocols.

\section{Statistical Methods}

The unpaired $t$ test was performed to compare differences between control and treated groups using GraphPad from Prism (version 6.0).

\section{RESULTS}

\section{Uptake of $6 "-18 \mathrm{~F}$-Fluoromaltotriose in Bacterial Cultures}

$6 "{ }^{\prime 1}{ }^{18}$ F-fluoromaltotriose (Supplemental Fig. 1 shows the structure) was incubated with different strains of bacteria (Fig. 1A). As shown previously for our first-generation tracer (14), 6" - ${ }^{18} \mathrm{~F}$-fluoromaltotriose was taken up by both gram-negative (E. coli and $P$. aeruginosa) and gram-positive ( $S$. aureus and L. monocytogenes) bacteria. The different strains of bacteria took up different amounts of $6 "{ }^{11} \mathrm{~F}$-fluoromaltotriose per cell, possibly reflecting the different expression levels of the maltodextrin transporter as well as the physical size of the bacterial cell. The uptake of $6 "-{ }^{18}$ F-fluoromaltotriose was blocked by coincubation with $1 \mathrm{mM}$ of maltose, indicating that the tracer was being taken up through the maltose transport system (Gayatri Gowrishankar and Mohammad Namavari, unpublished data, August 22, 2014). 6" -18F-fluoromaltotriose was not taken up by 2 mammalian cancer cell lines (Fig. 1C), as was also shown for the first-generation maltose tracer (14). In contrast to our first-generation disaccharide tracer, the new $6{ }^{\prime \prime}-{ }^{18} \mathrm{~F}-$ fluoromaltotriose tracer was retained well in E. coli cultures $(50 \%$ retention vs. 5\% retention; Supplemental Fig. 2).

\section{6"-18F-Fluoromaltotriose in E. Coli-Induced Myositis in Living Mice}

In a simple $E$. coli-induced myositis model, $6{ }^{\prime \prime}-{ }^{18} \mathrm{~F}$-fluoromaltotriose was able to distinguish infected muscle from the uninfected contralateral muscle (3.4-fold, $P<0.05$; Fig. 1B). $6{ }^{\prime \prime}-18$ F-fluoromaltotriose was also able to detect as little as $10^{6}$ CFUs of E. coli in mice (Fig. 2B). The tracer is taken up only in viable bacteria (shown by yellow arrow), as is its predecessor (Fig. 2B). There appears to be a modest correlation between the amount of tracer that was taken up and the number of implanted E. coli

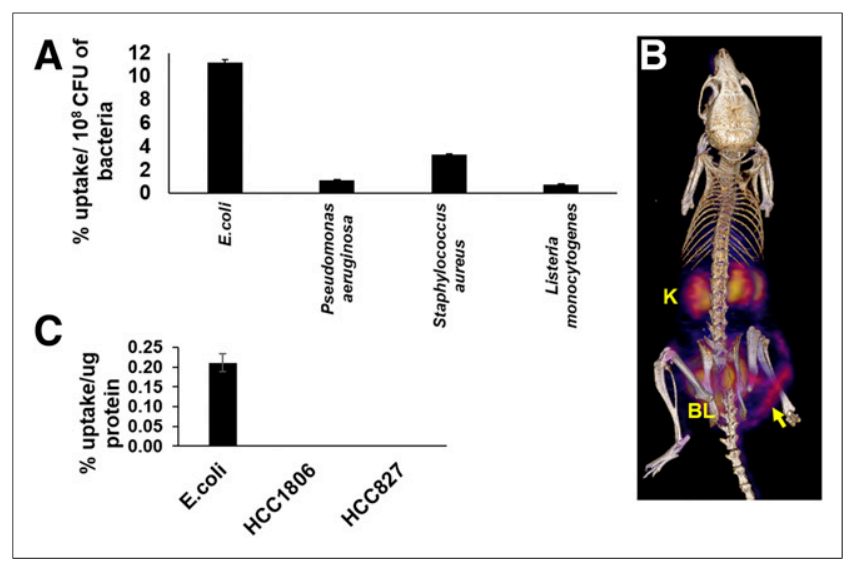

FIGURE 1. Evaluation of 6"-18 F-fluoromaltotriose in bacterial cultures and in mice. (A) Uptake of $66^{\prime \prime}-18 \mathrm{~F}$-fluoromaltotriose in indicated strains of bacteria for $30 \mathrm{~min}$. (B) Three-dimensional color map from small-animal $\mathrm{PET} / \mathrm{CT}$ scan of representative nude mouse with $E$. coli infection in its right thigh $24 \mathrm{~h}$ after infection and $1 \mathrm{~h}$ after intravenous injection of 7.4 $\mathrm{MBq}$ of $6^{\prime \prime}-{ }^{18} \mathrm{~F}$ fluoromaltotriose. Also seen in image is bladder (BL) and kidneys (K). Yellow arrow highlights site of infection. (C) Uptake of $66^{\prime \prime}{ }^{18} \mathrm{~F}-$ fluoromaltotriose in E. coli versus mammalian cancer cell lines HCC1806 and HCC827. Error bars are SD of means of triplicate samples.

(Pearson $r=0.61, P<0.05$; Supplemental Fig. 3). Dynamic PET/ CT scans acquired over the course of an hour showed excellent retention of $6 "-{ }^{18} \mathrm{~F}$-fluoromaltotriose in the infected muscle (Fig. $2 \mathrm{~A})$ in an E. coli-induced myositis model, with the maximum accumulation seen at $30 \mathrm{~min}$ after tracer administration. Ex vivo biodistribution studies comparing $6-{ }^{18} \mathrm{~F}$-fluoromaltose and $6{ }^{1}-{ }^{18} \mathrm{~F}$ fluoromaltotriose showed a predominantly renal clearance, with some clearance via the hepatobiliary system (Fig. 2C). $6{ }^{11}-{ }^{18} \mathrm{~F}-$ fluoromaltotriose was not taken up differentially in an LPSinduced lung inflammation model (1.04-fold difference between lungs of control and LPS-treated mice; Figs. 3A and 3B), confirming its specificity for imaging bacterial infection.

\section{6"-18 F-Fluoromaltotriose in a P. Aeruginosa Wound Infection Model}

$P$. aeruginosa is a well-known pathogen that often infects wounds and burns. It has also been notoriously difficult to image. In an effort to replicate a clinically relevant wound infection model, $10^{6}$ CFUs of Xen 5, a bioluminescent strain of $P$. aerugi$n o s a$, were inoculated into a superficial wound made into the back of immunocompetent mice. Twenty-four hours later, bioluminescent images of the mice were obtained, confirming the presence of viable bacteria (Fig. 4A). Small-animal PET/CT images obtained within $2 \mathrm{~h}$ (Fig. 4B) showed the accumulation of $6{ }^{\prime \prime}{ }^{18}$ F-fluoromaltotriose in the infected wounds and little to no accumulation in the control mice wounds with phosphate-buffered saline administration. Ex vivo biodistribution studies demonstrated a significant difference between infected and control wounds (4-fold difference, $P<0.05$; Supplemental Fig. 4).

\section{6"-18 F-Fluoromaltotriose in Incidental S. Aureus Infection Model}

A nude rat that developed an incidental bacterial infection after cardiac surgery was imaged by small-animal PET/CT after the administration of $6 "{ }^{18}$ F-fluoromaltotriose via the tail vein. Necropsy of a similarly infected rat from the same group revealed extensive botryomycosis-like lesions due to $S$. aureus (data not shown). Botryomycosis is a bacterial infection characterized by 


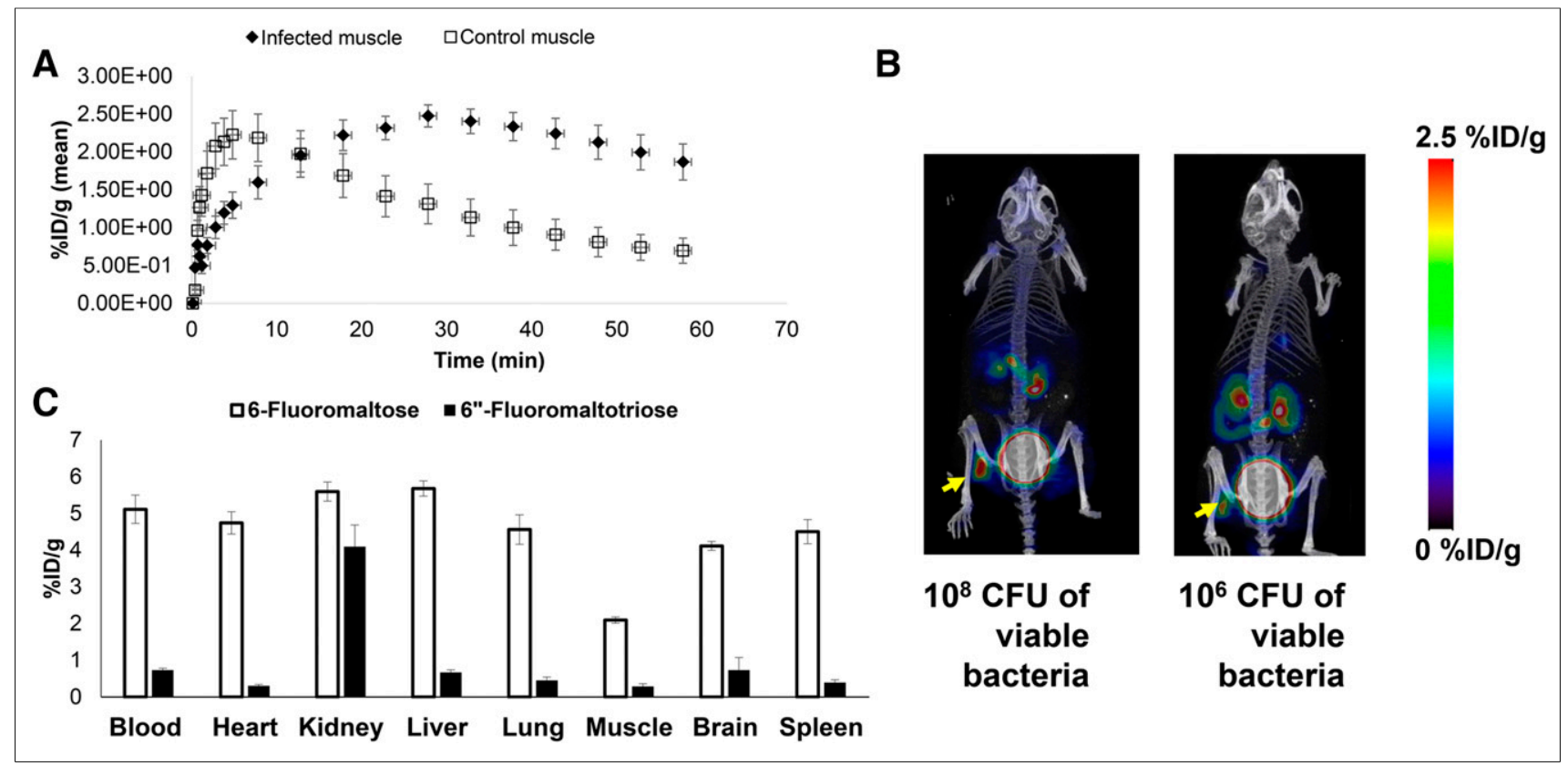

FIGURE 2. Evaluation of $6 "-18 \mathrm{~F}$-fluoromaltotriose in $E$. coli-induced myositis. (A) Time-activity curve from 1-h dynamic small-animal PET/CT scan of mice $(n=4)$ showing accumulation of $6^{\prime \prime}-18 \mathrm{~F}$-fluoromaltotriose in E. coli-infected muscle. (B) Maximum-intensity projections from small-animal $\mathrm{PET} / \mathrm{CT}$ of representative mice bearing $10^{8}$ and $10^{6} \mathrm{CFUs}$ of viable $E$. coli in left muscle and same number of heat-inactivated bacteria in right muscle, $1 \mathrm{~h}$ after intravenous administration of tracer. (C) Ex vivo biodistribution comparing 6- ${ }^{18} \mathrm{~F}-$-fluoromaltose (light gray bars) $(n=3)$ and 6 "- $-18 \mathrm{~F}-$ fluoromaltotriose (black bars) $(n=3)$ in nude mice, $1 \mathrm{~h}$ after administration of tracer. Error bars represent SD of means. \%ID/g = percentage injected dose per gram.

granulomatous skin lesions filled with pus and bacteria. A 60-min dynamic PET/CT scan was acquired, revealing focal uptake of $6 "-{ }^{18} \mathrm{~F}$-fluoromaltotriose in the thoracic region (Fig. 5, left), which was absent in the control rats (Fig. 5, right). The rat was then treated with Cefazolin (Glaxo Smith Kline), a broad-spectrum antibiotic, for $1 \mathrm{mo}$ and then imaged again on the small-animal PET/CT scanner after tail vein injection of $6^{\prime \prime}-{ }^{18} \mathrm{~F}$-fluoromaltotriose. The PET images obtained after antibiotic therapy show a marked reduction in the uptake of the tracer in the thoracic region (Fig. 5, middle), confirming that the initial uptake was likely due to the presence of a bacterial infection.

\section{DISCUSSION}

In this study, we present data characterizing $6 "{ }^{\prime 1}{ }^{18} \mathrm{~F}$-fluoromaltotriose, a novel tracer targeting the maltodextrin transporter of

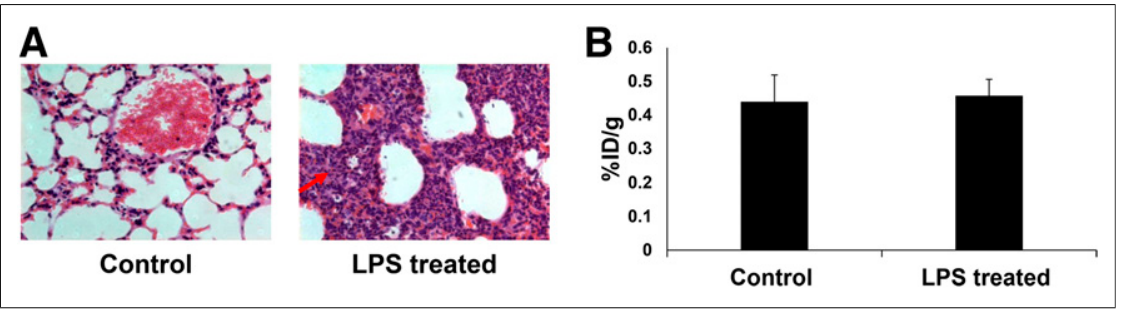

FIGURE 3. Evaluation of 6"-18F-fluoromaltotriose in inflammation. (A) Representative hematoxylin and eosin-stained sections of formalin-fixed lungs from control mice (left) and from mice with LPS-induced lung inflammation (right) showing thickening of bronchial walls (red arrow) due to accumulation of neutrophils and macrophages (stained blue). (B) Ex vivo biodistribution quantitation of lungs from immunocompetent control mice $(n=5)$ and mice after LPS-induced lung inflammation $(n=5), 24 \mathrm{~h}$ after administration of LPS intranasally and $1 \mathrm{~h}$ after intravenous administration of $6 "-18$ F-fluoromaltotriose. Error bars represent SD of means. bacteria (Supplemental Fig. 8). 6" ${ }^{18}$ F-fluoromaltotriose is a second-generation tracer and displayed a marked improvement in its pharmacokinetic profile over the first-generation tracer, as shown in Figure 2C, undergoing predominantly renal clearance. This result is in contrast to our first-generation tracer, 6- ${ }^{18} \mathrm{~F}$-fluoromaltose, which displayed extensive hepatobiliary clearance (Fig. 2C) (14). A head-to-head comparison of the biodistribution profiles of $6-{ }^{18} \mathrm{~F}$-fluoromaltose and $6 "{ }^{18} \mathrm{~F}$-fluoromaltotriose (Fig. 2C) showed that the maltotriose tracer also had reduced uptake in blood and muscle as compared with the maltose tracer, thereby boosting the target-to-background ratios significantly. The $6{ }^{\prime \prime}{ }^{18} \mathrm{~F}-$ fluoromaltotriose tracer showed improved retention in E. coli, as shown in Supplemental Figure 2 (50\% vs. 5\% for the $6-{ }^{18}$ F-fluoromaltose) (14), indicating that perhaps the maltotriose is being metabolized to glucose at a slower rate than the maltose. This difference in metabolism could also permit the $6^{\prime \prime}-{ }^{18} \mathrm{~F}$-fluoromaltotriose to discriminate between bacterial and fungal infections. There is evidence that, although fungi can take up and metabolize maltose, maltotriose appears unique in that it is metabolized more slowly in yeast (25). More experiments with bacteria bearing mutations in the maltodextrin transporter are needed to truly understand the mechanism of uptake and use of the tracer.

The improved pharmacokinetic profile also leads to an improvement in sensitivity. As shown in Figure 2B, $6{ }^{\prime \prime}{ }^{18} \mathrm{~F}$-fluoromaltotriose can pick up as few as $10^{6} \mathrm{CFUs}$ of $E$. coli (infected tissues usually have $10^{5}-10^{6}$ CFUs 


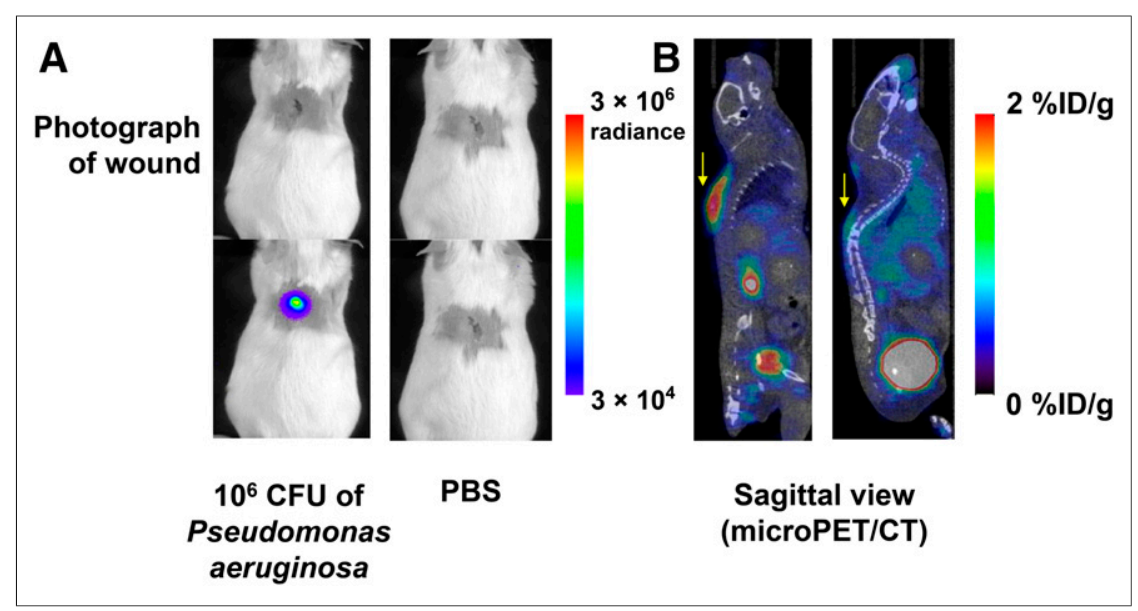

FIGURE 4. Evaluation of $6^{\prime \prime 18} \mathrm{~F}$-fluoromaltotriose in $P$. aeruginosa wound infection model. (A) Bioluminescence images of CD1 mice bearing $P$. aeruginosa-infected wound (left) and control mice (right). (B) Sagittal slices from small-animal PET/CT scan of same mice $1 \mathrm{~h}$ after intravenous administration of $6 "-18$ F-fluoromaltotriose

of bacteria), with an improved signal-to-noise ratio as compared with the first-generation tracer $6-{ }^{18} \mathrm{~F}$-fluoromaltose $(14)$. There is also evidence that tracers targeting the maltodextrin transporter can be taken up in $S$. aureus biofilms, as shown by Murthy et al. (16). This class of imaging agents can therefore be used to image bacterial biofilm infections. Although there has been a report on the use of ${ }^{18} \mathrm{~F}-\mathrm{FDG}$ to image $S$. aureus biofilms in mice (26), the use of $6 "{ }^{18} \mathrm{~F}$-fluoromaltotriose would enable imaging the biofilm infection rather than the surrounding inflammation. Bacterial biofilms have been implicated in the pathogenesis of a wide variety of chronic infections, particularly in patients who have had surgical implants or prosthetics (27). Other instances of chronic infections include cystic fibrosis patients who have lung infections often attributed to $P$. aeruginosa biofilms (28). Our data show that $6 "-{ }^{18} \mathrm{~F}$ fluoromaltotriose is taken up by $P$. aeruginosa both in vitro and in vivo in a wound infection model, and it is likely that the tracer would be taken up in $P$. aeruginosa biofilms, which we hope to explore in the future. Additionally, this is the only report we know of in which an imaging agent is shown to be capable of detecting $P$. aeruginosa infections in vivo (both ${ }^{124}$ I-FIAU (9) and fluorodeoxysorbitol (13) were not taken up by P. aeruginosa).

Maltotriose does not cross the bloodbrain barrier, so it cannot be used for imaging bacterial meningitis, nor can it be used to image infections caused by certain bacterial pathogens that may have an intracellular phase in their life cycle (e.g., $L$. monocytogenes, Salmonella typhimurium, Mycobacterium tuberculosis). In the case of $L$. monocytogenes, we have observed in an in vivo infection model (Supplemental

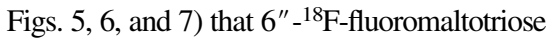
was able to detect the infection in mice that appeared to have widespread disseminated systemic infection, as observed by bioluminescence imaging (Supplemental Figs. 5 and 6), and elevated uptake in blood (Supplemental Fig. 7), which has been observed in a small percentage of infected animals in other studies (29). It may therefore be possible to image facultative intracellular pathogens such as $M$. tuberculosis, using $6 "{ }^{18} \mathrm{~F}$ - fluoromaltotriose if some of the bacteria are extracellular. However, extensive studies would be required to explore such a possibility. In many ways, $6{ }^{\prime \prime}-{ }^{18} \mathrm{~F}-$ fluoromaltotriose (and other tracers targeting the maltodextrin transporter) are conceptually similar to ${ }^{18} \mathrm{~F}$-FDG. Just as ${ }^{18} \mathrm{~F}-\mathrm{FDG}$ is internalized into cells and used in the diagnosis of most human cancers, $6 "{ }^{18} \mathrm{~F}$ - fluoromaltotriose is internalized in several bacterial pathogens, both gram-negative and gram-positive (Fig. 1A). Also, just as ${ }^{18} \mathrm{~F}$-FDG cannot distinguish between different types of cancers, $6 "{ }^{18} \mathrm{~F}$-fluoromaltotriose cannot distinguish between different types of bacterial strains. Tracers under investigation, such as ${ }^{18} \mathrm{~F}$ fluorodeoxysorbitol and others (30), which are internalized by specific bacterial pathogens, might be used in conjunction to complete

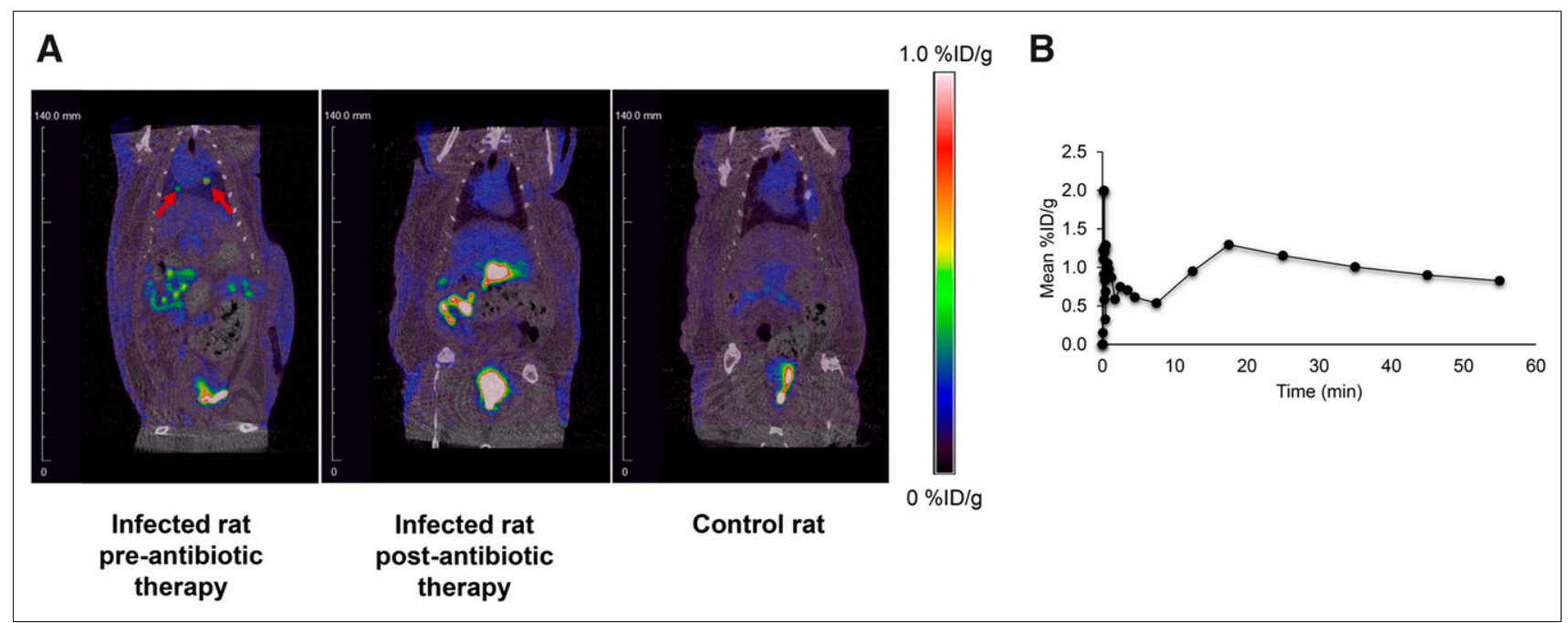

FIGURE 5. Evaluation of $6 "-18 \mathrm{~F}-$ fluoromaltotriose PET/CT in rat with incidental infection after cardiac surgery before and after antibiotic therapy. (A) $6^{\prime \prime}-{ }^{18} \mathrm{~F}$-fluoromaltotriose small-animal PET/CT images of rat before (left) and after $1 \mathrm{mo}$ of antibiotic treatment (middle). Image was taken $1 \mathrm{~h}$ after intravenous administration of $6 "-{ }^{18} \mathrm{~F}$-fluoromaltotriose. Right panel shows control rat that also received tracer. There is virtually no uptake near heart in control rat. (B) Time-activity curve of hottest lesion in infected rat before antibiotic therapy. $\% \mathrm{ID} / \mathrm{g}=$ percentage injected dose per gram. 
the diagnosis when clinically necessary. In contrast to ${ }^{18} \mathrm{~F}-\mathrm{FDG}$, however, $6 "{ }^{18} \mathrm{~F}$-fluoromaltotriose can apparently distinguish infection from inflammation (Fig. 3B).

The study performed in rats (Fig. 5) confirms the pharmacokinetic profile observed in mice (predominantly renal), and also illustrates the potential utility of $6 "{ }^{\prime 18} \mathrm{~F}$-fluoromaltotriose in monitoring responses to antibiotic therapy. The study also reflects the clinically relevant situation in which a severe infection must be treated and monitored without definitive identification of the pathogen. Although it is likely that the rat in question was infected with $S$. aureus as in the other similarly infected rat in the same group, this assumption was not verified and yet the imaging proved efficacious. Given the rise in antibiotic resistance, the use of imaging to guide the choice and duration of antibiotic treatment can help mitigate the emergence of resistance. This possibility becomes important in diseases such as tuberculosis and osteomyelitis, which involve prolonged use of antibiotics, because physicians may be unable to determine whether the bacterial infection has been successfully treated. Although PET itself cannot replace emergent point-of-care diagnostics as a screening tool for infectious diseases, $6{ }^{\prime \prime}{ }^{18} \mathrm{~F}$-fluoromaltotriose and other similar PET tracers in development will play a key role in the hospital setting to confirm, localize, and determine the spread of bacterial infection, particularly in the context of other confounding indications such as inflammation and cancer.

\section{CONCLUSION}

$6 "{ }^{18} \mathrm{~F}$-fluoromaltotriose is the second in a series of tracers that are being developed targeting the bacterial maltodextrin transporter. The studies outlined above have demonstrated that the tracer is able to image bacterial infections in preclinical models and shown that the pharmacokinetic properties of this novel tracer make it suitable for future clinical studies. The tracer cannot be used to image infections caused by bacteria that invade cells, for example, M. tuberculosis, thus limiting the utility of the tracer.

\section{DISCLOSURE}

Part of the study was supported by the following grants: CIRM RT3-07798, CIRM DR2-05394, NIH R01 HL133272, and NIH T32 EB009035. The authors have a patent on $6 "{ }^{18} \mathrm{~F}$-fluoromaltotriose (Probes and Methods of Imaging a Bacterial Infection; serial no., 15/096,308; filing date, April 12, 2016). No other potential conflict of interest relevant to this article was reported.

\section{ACKNOWLEDGMENTS}

We acknowledge Dr. Frezghi Habte and the Stanford University Small Animal Imaging Facility for their help with the image analysis and quantitation. We are also grateful to the radiochemistry facility for their radiochemistry support. Moreover, we are deeply appreciative for the funding support from our grants.

\section{REFERENCES}

1. Marston HD, Dixon DM, Knisely JM, Palmore TN, Fauci AS. Antimicrobial resistance. JAMA. 2016;316:1193-1204.

2. Morens DM, Fauci AS. Emerging infectious diseases: threats to human health and global stability. PLoS Pathog. 2013;9:e1003467.

3. Antibiotic resistant threats in the United States. Centers for Disease Control and Prevention website. https://www.cdc.gov/drugresistance/threat-report-2013/. Updated April 10, 2017. Accessed August 25, 2017.
4. National action plan for combating antibiotic resistant bacteria. Centers for Disease Control and Prevention website. https://www.cdc.gov/drugresistance/pdf/national_ action_plan_for_combating_antibotic-resistant_bacteria.pdf. March 2015. Accessed August 25, 2017

5. Janda JM, Abbott SL. 16S rRNA gene sequencing for bacterial identification in the diagnostic laboratory: pluses, perils, and pitfalls. J Clin Microbiol. 2007;45: 2761-2764.

6. Sasser TA, Van Avermaete AE, White A, et al. Bacterial infection probes and imaging strategies in clinical nuclear medicine and preclinical molecular imaging. Curr Top Med Chem. 2013;13:479-487.

7. van Oosten M, Hahn M, Crane LM, et al. Targeted imaging of bacterial infections: advances, hurdles and hopes. FEMS Microbiol Rev. 2015;39:892-916.

8. Bunschoten A, Welling MM, Termaat MF, Sathekge M, van Leeuwen FW. Development and prospects of dedicated tracers for the molecular imaging of bacterial infections. Bioconjug Chem. 2013;24:1971-1989.

9. Peterson KL, Reid WC, Freeman AF, et al. The use of ${ }^{14} \mathrm{C}$-FIAU to predict bacterial thymidine kinase presence: implications for radiolabeled FIAU bacterial imaging. Nucl Med Biol. 2013;40:638-642.

10. Diaz LA Jr, Foss CA, Thornton K, et al. Imaging of musculoskeletal bacterial infections by [ ${ }^{124}$ I]FIAU-PET/CT. PLoS One. 2007;2:e1007.

11. Ebenhan T, Zeevaart JR, Venter JD, et al. Preclinical evaluation of ${ }^{68} \mathrm{Ga}$-labeled 1,4,7-triazacyclononane-1,4,7-triacetic acid-ubiquicidin as a radioligand for PET infection imaging. J Nucl Med. 2014;55:308-314.

12. Vilche M, Reyes AL, Vasilskis E, Oliver P, Balter H, Engler H. ${ }^{68}$ Ga-NOTAUBI-29-41 as a PET tracer for detection of bacterial infection. J Nucl Med. 2016;57:622-627.

13. Weinstein EA, Ordonez AA, DeMarco VP, et al. Imaging Enterobacteriaceae infection in vivo with ${ }^{18} \mathrm{~F}$-fluorodeoxysorbitol positron emission tomography. Sci Transl Med. 2014;6:259ra146.

14. Gowrishankar G, Namavari M, Jouannot EB, et al. Investigation of $6-\left[{ }^{18} \mathrm{~F}\right]-$ fluoromaltose as a novel PET tracer for imaging bacterial infection. PLoS One. 2014;9:e107951.

15. Namavari M, Gowrishankar G, Hoehne A, Jouannot E, Gambhir SS. Synthesis of $\left[{ }^{18} \mathrm{~F}\right]$-labelled maltose derivatives as PET tracers for imaging bacterial infection. Mol Imaging Biol. 2015;17:168-176.

16. Ning X, Lee S, Wang Z, et al. Maltodextrin-based imaging probes detect bacteria in vivo with high sensitivity and specificity. Nat Mater. 2011;10:602-607.

17. Ferenci T. The recognition of maltodextrins by Escherichia coli. Eur J Biochem. 1980;108:631-636.

18. Gopal S, Berg D, Hagen N, et al. Maltose and maltodextrin utilization by Listeria monocytogenes depend on an inducible $\mathrm{ABC}$ transporter which is repressed by glucose. PLoS One. 2010;5:e10349.

19. Hanses F, Roux C, Dunman PM, Salzberger B, Lee JC. Staphylococcus aureus gene expression in a rat model of infective endocarditis. Genome Med. 2014;6:93-109.

20. Jones SA, Jorgensen M, Chowdhury FZ, et al. Glycogen and maltose utilization by Escherichia coli O157:H7 in the mouse intestine. Infect Immun. 2008;76: 2531-2540.

21. Lång $\mathrm{H}$, Jonson $\mathrm{G}$, Holmgren $\mathrm{J}$, Palva ET. The maltose regulon of Vibrio cholerae affects production and secretion of virulence factors. Infect Immun. 1994;62:4781-4788.

22. Ferenci T. Methyl-alpha-maltoside and 5-thiomaltose: analogs transported by the Escherichia coli maltose transport system. J Bacteriol. 1980;144:7-11.

23. Ning X, Seo W, Lee S, et al. PET imaging of bacterial infections with fluorine18-labeled maltohexaose. Angew Chem Int Ed Engl. 2014;53:14096-14101.

24. Shelburne SA, 3rd, Sumby P, Sitkiewicz I, et al. Maltodextrin utilization plays a key role in the ability of group A Streptococcus to colonize the oropharynx. Infect Immun. 2006;74:4605-4614.

25. Zastrow CR, Hollatz C, de Araujo PS, Stambuk BU. Maltotriose fermentation by Saccharomyces cerevisiae. J Ind Microbiol Biotechnol. 2001;27:34-38.

26. Garrido V, Collantes M, Barberan M, et al. In vivo monitoring of Staphylococcus aureus biofilm infections and antimicrobial therapy by $\left[{ }^{18} \mathrm{~F}\right]$ fluoro-deoxyglucoseMicroPET in a mouse model. Antimicrob Agents Chemother. 2014;58:66606667.

27. Bjarnsholt T, Alhede M, Alhede M, et al. The in vivo biofilm. Trends Microbiol. 2013;21:466-474.

28. Trøstrup H, Thomsen K, Christophersen LJ, et al. Pseudomonas aeruginosa biofilm aggravates skin inflammatory response in BALB/c mice in a novel chronic wound model. Wound Repair Regen. 2013;21:292-299.

29. Merrick JC, Edelson BT, Bhardwaj V, Swanson PE, Unanue ER. Lymphocyte apoptosis during early phase of Listeria infection in mice. Am J Pathol. 1997;151: 785-792.

30. Ordonez AA, Weinstein EA, Bambarger LE, et al. A systematic approach for developing bacteria-specific imaging tracers. J Nucl Med. 2017;58:144-150. 